\title{
ACCIDENTS OF MOTORCYCLISTS AGAINST ROADSIDE INFRASTRUCTURE
}

Francisco J. López-Valdés, David García, David Pedrero and José L. Moreno CIDAUT, Parque Tecnológico de Boecillo, P. 209, 47151 Boecillo, Valladolid, Spain; Phone: +34983 548035, Fax: +34983 548062, E-mail:fralop@,cidaut.es

\begin{abstract}
Powered two wheelers (PTWs) riders are one of the most vulnerable groups of road users. Roadside barriers work quite effectively for passenger cars, but sometimes they are dangerous for motorcyclists, especially guard-rails. These accidents are investigated by means of an analysis of the DGT Spanish National Accident Database and reviewing different in-depth studies. Motorcyclist injury patterns have been identified. A revision of the injury criteria used for head and neck injuries has been carried out to select those suitable to be used in an impact of a motorcyclist against a roadside barrier. Head injury criterion (HIC36) for head injury has been accepted as a valid injury criterion. Regarding the neck, the Mertz criterion was considered in a first stage and some changes have been introduced in order to suit the peculiarities of motorcyclists' impacts, using adapted methods from the automobile sector related to Out-of-position (OOP) scaling methodology.
\end{abstract}

Key words: motorcyclists accidents, guard-rails, head injury criteria, neck injury criteria.

\section{INTRODUCTION}

In Spain, the number of motorcycles and moped has grown continuously from 2000 to 2002 due to their advantages as flexibility or enjoyment. However, these vehicles are often involved in accidents and they account for a large number of casualties. For example, in 2002, 784 motorcyclists died in road accidents in Spain (both motorcycle and moped riders). This figure implies that the $14.7 \%$ of the total number of casualties were riders, while

Michael D. Gilchrist (ed.), IUTAM Proceedings on Impact Biomechanics: From Fundamental Insights to Applications, 163-170.

(C) 2005 Springer. Printed in the Netherlands. 
the total number of powered two wheels vehicles (PTW) was the $13.3 \%$ of the total number of registered vehicles in the country.

Moreover, it also should be pointed that, although the number of cars has also increased during those years, the number of accidents of passenger cars has decreased but when focusing on motorcycles, the number of accidents in which a motorcycle is involved has even increased in the period studied.

Similar trends have been found in other different countries in Europe as The Netherlands, Italy or Germany [1].

\section{ACCIDENTOLOGY STUDIES: INJURY PATTERNS}

\subsection{Spanish National Road Accident Database}

Accident data from Spain have been extracted from the Spanish National Road Accident Database, intensive database performed by a public organism called DGT (General Directorate of Transport), depending on the Ministry of Interior. It contains every road accident with casualties in Spanish roads and is performed by Police forces at the scene of the accident.

In 2002, 5,979 motorcycle/moped accidents occurred in Spanish roads where 7,834 road users resulted injured. Nearly $20 \%$ of these accidents were run off the road accidents, in which the likelihood of the impact of the rider against roadside infrastructure or against a hazardous object placed close to the carriageway is extremely high. Barriers have demonstrated their effectiveness restraining passenger cars or heavier vehicles but, due to the unrestrained position of the rider and the peculiarities of motorcycle accidents, they could be dangerous as riders usually impact against the barrier with their own body. Special attention must be paid to metal guardrails and posts.

In a first stage of this study, data from the Spanish national database were analyzed in order to obtain the magnitude of the problem. However, two difficulties arise from this analysis. The first one is identifying if the motorcyclist has hit the metal barrier or not when the accident occurs using this intensive database. The second one is related to injury codification done by the Police at the moment of the accident. It can be assigned only one type of injury to each casualty, trying to code the most serious one. Sometimes this codification is not trivial and medical knowledge should be required.

Only injuries sustained by riders involved in accidents outside of urban areas have been studied, as the likelihood of hitting a barrier is higher. 


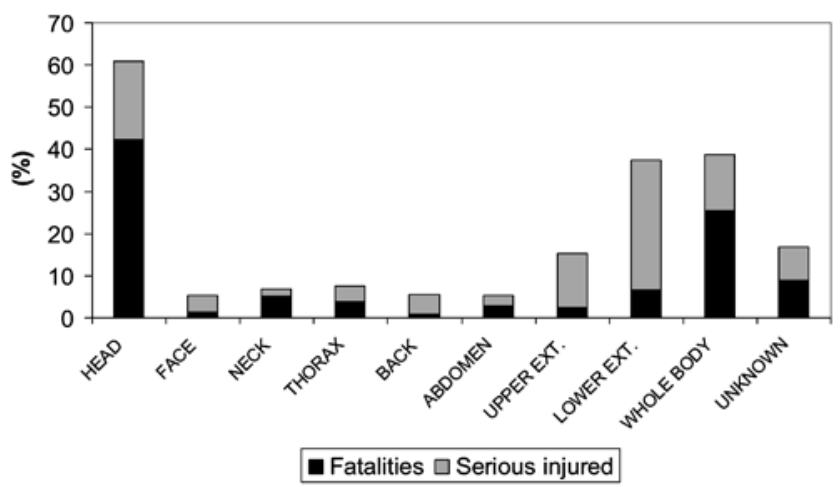

Figure 1. Main injured motorcyclist body region in fatal and serious accidents in non-urban areas. Spain, 2002.

Figure 1 shows injuries sustained by fatalities and serious injured riders. Head, extremities and whole body injuries are the most frequent injured body region.

However, it is necessary to analyze in-depth data to obtain a better insight of the sustained injuries. Available in-depth information from European and American sources has been reviewed.

\subsection{In-Depth Accident Studies}

Hell and Lob [2] studied 173 motorcyclist accidents in Munich from 1985 to 1990. The study considered all accident configurations. In 27 cases, riders hit a fixed object (e.g. a tree, a post, a metal barrier).

Table 1. Injured body regions (AIS2+). Motorcyclists vs. fixed objects. Munich, 1985-1990.

\begin{tabular}{ll}
\hline Body region & Frequency \\
\hline Head & $63 \%$ \\
Thorax & $48 \%$ \\
Abdomen & $19 \%$ \\
Pelvis & $7 \%$ \\
Spinal cord & $33 \%$ \\
Upper extremities & $37 \%$ \\
Lower extremities & $37 \%$ \\
\hline
\end{tabular}

These accidents were found to be the most sever (39 fatalities) within the studied sample. It should be pointed again that this data are not focus only in impacts against barriers but also against other objects. Again, special role is played by head and extremities, although it is also found the importance of thorax and back injuries (which are more difficult to be identified by the 
Police and need a better insight into the casualties performed by medical teams). The internationally accepted Abbreviated Injury Scale (AIS) defined by the Association for the Advancement of Automotive Medicine (AAAM) is used to define the severity of the injuries.

Hurt et al. [3] found that the frequency of AIS 3+ injuries of motorcyclists depending on the body region was distributed according percentages presented in Table 2, although in this case the information is not only focused on accidents against fixed objects:

Table 2. Injured body regions (AIS3+). Motorcyclists. California.

\begin{tabular}{ll}
\hline Body region & Frequency \\
\hline Head, neck and face & $32.3 \%$ \\
Thorax & $18.3 \%$ \\
Abdomen & $10.5 \%$ \\
Upper extremities & $15.5 \%$ \\
Lower extremities & $23.4 \%$ \\
\hline
\end{tabular}

Neck injuries are not specifically mentioned in these last studies. However, Thom et al. [4] pointed that fatal injuries to the neck are more frequent than most of the studies shown but they need to be carefully examined to be identified. They performed the autopsy to 304 dead motorcyclists and they found 195 dislocations and 28 fractures, all of them placed at the upper region of the spine (C1-C2 level). They did not found differences in wearing helmet or not in these neck injuries. Similar findings are reported by Bishop and Wells [5] and Orsay et al. [6].

However, one of the most important motorcyclist accidents in-depth studies was the mentioned study performed by Hurt et al. [7] in California. From the accidents studied, the most severe neck injury was only a minor cervical sprain or complaint of pain. They suggested that as long as usually the load to the neck is transmitted from the head, the lower the forces on the head the lower the load on the neck.

At last, Otte [8] studied 876 motorcycle accidents from 1985 to 1995 in Hannover. He reported that head and lower extremities serious injuries are frequent in accidents against fix objects.

These in-depth accident studies are not focused on impacts against roadside infrastructure. Two investigations have been considered, focused specifically on these accidents. The first one has been performed by the Aerospace Engineering Department of the University of Milan [9]. This study focuses mainly on head, thorax, abdomen and spinal cord injuries. The second, performed by the French institute INRETS, is addressed to develop a test procedure for impacts of motorcyclists against metal barriers, based on an accidentology study at the area of Lyon during 1995 [10]. Nevertheless, this accident investigation was not only focused on accidents against guard 
rails, but also other kind of impacts. At last, it was decided to consider only head and neck in the test, as they were the most severe injuries found.

In conclusion, after the revision of the available documentation and data from different sources, it was decided to review the biomechanical criteria that could be suitable to study the impact of a motorcyclist against roadside infrastructure. This review is focused only on head and neck criteria, as they are generally mentioned as the most important injuries that could cause a fatal accident in almost all the studies.

\section{HEAD INJURY CRITERION (HIC)}

The brain is one of the most critical body parts due to its importance and vulnerability. Hurt et al. [7] found in their study that the regions of concern were the cranium and the enclosed brain, both protected by the helmet. Only not penetrating injuries are considered in this study, as the impacts of motorcyclist against barriers used to be of this kind. It is proposed to use HIC (Head Injury Criterion) as criterion for this head impacts. This parameter was defined based on investigations performed at the Wayne State University [12]. It has been revised from 1960 to 1986, when it was established that the time period considered in the measures should be 36 milliseconds.

Although this criterion has been developed for frontal impacts and only considers linear accelerations, it is commonly accepted in some lateral impact protocols as in EuroNCAP, for instance. Thus, it is proposed that, in a first stage and without trying to develop new criteria focused only in riders, HIC criterion is valid to evaluate the injuries to the head in accidents of motorcyclists against metal barriers. It is assumed that the same level as it is established in the EuroNCAP protocol can be sustained by the motorcyclist:

$$
H I C_{36} \leq 1000 \Rightarrow p(\text { AIS } 3+)=20 \%
$$

However, it is necessary to consider that when testing this kind of impacts the helmet should be taken into account, so more considerations need to be done regarding the position and fixing of the helmet to the dummy. Moreover, as the aim of this paper is to give information that could be used in the development of a procedure to test impacts of motorcyclists against barriers using available tools, it is proposed that a conventional dummy should be used in the tests, so a method to attach the helmet to the head of the Hybrid III dummy has been also investigated. Further improvement of head injury criteria has been recently suggested in the project COST 327 [13], but there is not a clear established test procedure yet. 


\section{NECK INJURY CRITERIA}

European regulations and associations as EuroNCAP accept injury criteria for frontal impact based on the studies carried out by Mertz with slight modifications, considering $\mathrm{F}_{z}$ (axial force, both extension and compression), $\mathrm{F}_{\mathrm{x}}$ (shear force) and $\mathrm{M}_{\mathrm{y}}$ (both flexion and extension torques). These limits have been accepted for the case of motorcyclists. However, there is not any established limit for the other dynamic values $\mathrm{M}_{\mathrm{x}}, \mathrm{M}_{\mathrm{z}}$ and $\mathrm{F}_{\mathrm{y}}$, being the first of these three of great importance in side impact.

In USA, FMVSS 208 regulation for frontal impact uses the $\mathrm{N}_{\mathrm{ij}}$ criterion. Recently, this criterion has been also developed to be applied in Out-ofposition $(O O P)$ situations. OOP problematic is interesting for motorcyclists because several similarities can be found (mainly, dummy receiving a lateral impact in the head). A group of biomechanical experts of the Technical Working Group (TWG) has established the limits for the torques $\mathrm{M}_{\mathrm{x}}$ and $\mathrm{M}_{\mathrm{z}}$ based on their experience for these OOP situations [14]. OOP tests are performed using 3-years-old, 6-years-old, Hybrid III $5^{\text {th }}$ Small female and SID-IIs dummies, but not for Hybrid III $50^{\text {th }}$ male. Thus, $\mathrm{M}_{\mathrm{x}}$ values are not available for this dummy. To solve this inconvenience, it is proposed to use the same scaling method applied in frontal impact to obtain the $\mathrm{N}_{\mathrm{ij}}$ criterion for the other dummies of the Hybrid III family. This scaling process is based on geometrical factors and basically, the torques Hybrid III $50^{\text {th }}$ male are two times the torques in Hybrid III $5^{\text {th }}$ Small female [15].

In summary, values for $F_{x}, F_{z}$ and $M_{y}$ are extracted from EuroNCAP (mainly based on Mertz criteria). Value for $\mathrm{M}_{\mathrm{x}}$ is adapted from the OOP situation studied in FMVSS 208, scaling the proposed value for the Hybrid III $5^{\text {th }}$ Small female to the Hybrid III $50^{\text {th }}$ male. Values for $\mathrm{F}_{\mathrm{y}}$ and $\mathrm{M}_{\mathrm{z}}$ are not considered in this study. After this process, the proposed criterion is:

Table 3. Neck injury criterion.

\begin{tabular}{|c|c|c|c|c|c|}
\hline $\begin{array}{l}F_{x} \\
(N)\end{array}$ & $\begin{array}{l}\mathrm{F}_{\mathrm{z}} \text { tensile } \\
(\mathrm{N})\end{array}$ & $\begin{array}{l}\mathrm{F}_{\mathrm{z}} \text { compression } \\
(\mathrm{N})\end{array}$ & $\begin{array}{l}\mathrm{M}_{\mathrm{y}} \text { extension } \\
(\mathrm{Nm})\end{array}$ & $\begin{array}{l}M_{y} \text { flexion } \\
(\mathrm{Nm})\end{array}$ & $\begin{array}{l}\mathrm{M}_{\mathrm{x}} \\
(\mathrm{Nm})\end{array}$ \\
\hline Figure 2 & Figure 3 & Figure 4 & 57 & 190 & 134 \\
\hline
\end{tabular}

When all these values are respected, it is considered that the neck injury criterion is fulfilled. 


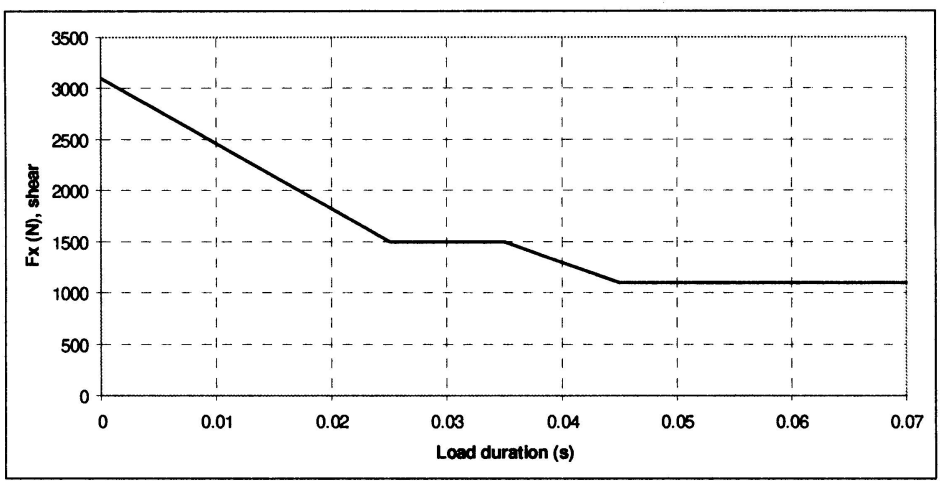

Figure 2. $F_{x}$ shear force.

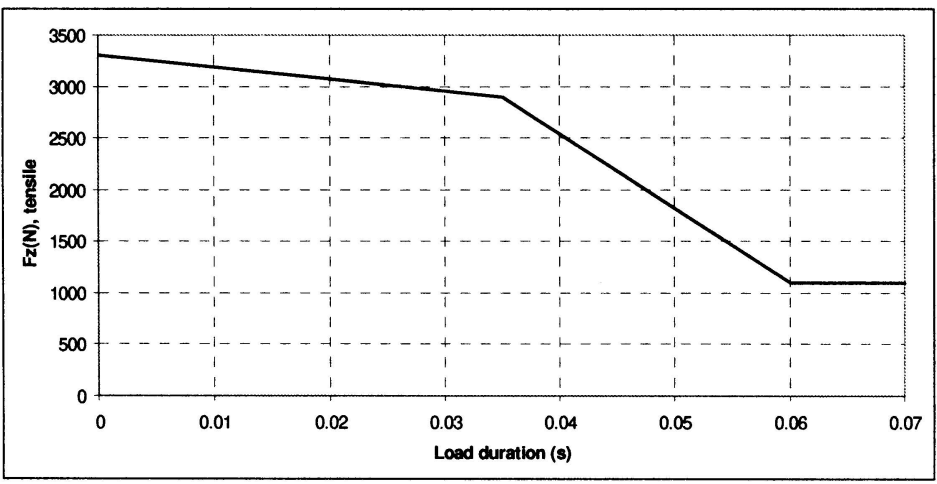

Figure 3. $F_{z}$ tensile force.

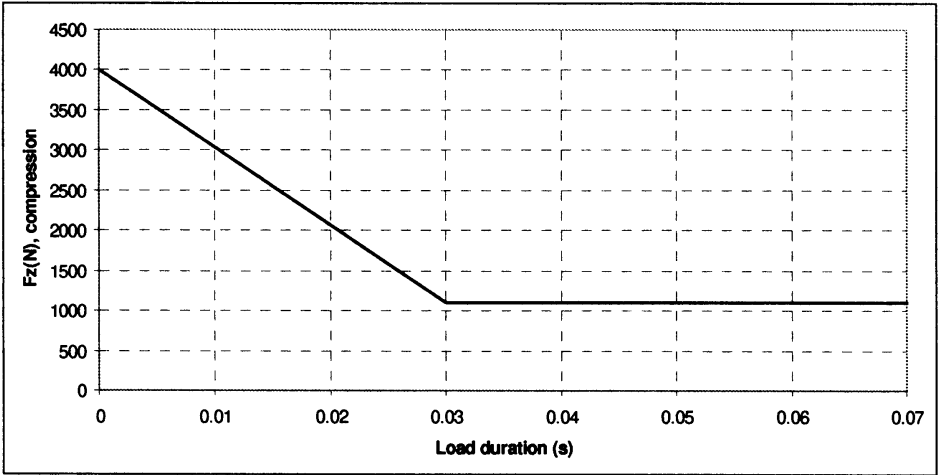

Figure 4. $\mathrm{F}_{\mathrm{z}}$ compression force. 


\section{CONCLUSION}

After the revision of the injury patterns of motorcyclists involved in accidents against barriers, injury criteria have been proposed for head and neck injuries.

They have been adapted from the automobile world and can be measured using a Hybrid III 50\% dummy, rather than trying to develop injury criteria only focused on riders and needing specific dummies. These criteria can be used in testing of barriers to improve the safety of roadside infrastructure considering also motorcyclists and not only cars.

\section{REFERENCES}

1. AP-SP41-0001. APROSYS Sub Project 4: Motorcycle Accidents. European funded project. TIP-CT-2004-506503.

2. Hell, W. and Lob, G., Typical injury patterns of motorcyclists in different crash types Effectiveness and improvements of countermeasures, in $37^{\text {th }}$ Annual Proccedings of the AAAM, Texas, USA, November, 1993.

3. Hurt, H., Ouelletm J.V. and Jennings G., Motorcycle crash bar effectiveness: A reevaluation using AIS-80, in AAAM Conference, 1984.

4. Thom, D.R., Hurt, H.H. Jr., Smith, T.A. and Rehman, I., Atlas and axis injuries in fatal motorcycle collisions, in AAAM Conference, 1995.

5. Bishop, P.J. and Wells, R.P., The inappropiateness of helmet drop tests in assessing neck protection in head first impacts, The American Journal of Sport Medicine, 18(2), 1990.

6. Orsay, E.M., Muelleman R.L., Peterson T.D., Jurisisc, D.H., Kosasih, J.B. and Levy, P., Motorcycle helmets and spinal injuries: Dispelling the myth, Annals of Emergency Medicine, 23(4), 1994.

7. Hurt, H.H. Jr. and Ouellet, J.V., Effectiveness of motorcycle safety helmets and protective clothing, in Proceedings of the 25th Conference of the AAAM, San Francisco, California, USA, 1981.

8. Otte D., Benefit of in-depth data for analyzing injury mechanisms of accidents with bicyclists and motorcyclists, International Journal of Crashworthiness, 3(1), 1998.

9. Sala, G. and Astori, P., New concepts and materials for passive safety of motorcyclists, in IRCOBI Conference, 1998.

10. Quincy, R., Protocole d'essais de dispositif de retenue assurant la securite des motorcyclists, Laboratorie d'essais Inrets Equipments de la Route (LIER), 1998.

11. Chou C., Nyquist G., Analytical studies of the head injury criterion (HIC), SAE Paper No. 740082 .

12. Chinn, B., Canaple B., Derler, S., Doyle, D., Otte, D., Schuller, E. and Willinger, R., COST 327: Motorcycle Safety Helmets (Final report of the action), Belgium, 2001.

13. Lund, A.K., Recommended procedures for evaluating occupant injury risk from deploying side airbags, IIHS, August 2000.

14. Frontal Offset Crashworthiness evaluation: guidelines for rating injury measures, IIHS, May 2001. 